\title{
Phytochemical and Biological Progress of Ixeris sonchifoia (Bge.) Hance
}

\author{
Tingyu Wen, ${ }^{a}$ Fang Li, ${ }^{a}$ and Jifeng Liu ${ }^{*, a, b}$ \\ ${ }^{a}$ School of Pharmaceutical Sciences, Institute of Drug Discovery and Development, Key Laboratory of \\ Advanced Pharmaceutical Technology, Ministry of Education of China, Zhengzhou University, \\ Zhengzhou 450001, China \\ ${ }^{b}$ Collaborative Innovation Center of New Drug Research and Safety Evaluation, Zhengzhou, \\ Henan 450001, China \\ Email: liujf2009y@126.com (J. L.)
}

\begin{abstract}
Ixeris sonchifoia (Bge.) Hance is a famous traditional Chinese herb (TCH) for medicinal treatment and diet with wide distribution and rich resources. Flavonoids, triterpenoids and sesquiterpene lactones are the characteristic constituents of this $\mathrm{TCH}$. Biological investigation on I. sonchifoia revealed the remarkable pharmacological activities of this herb for the treatment of cardiovascular and cerebrovascular diseases. This review summarized the phytochemical and biological progress of $I$. sonchifoia to provide a reference for further research and development of this $\mathrm{TCH}$.
\end{abstract}

Keywords Ixeris sonchifoia, chemical composition, pharmacological activities, flavonoids, sesquiterpene lactones, anti-tumor

\section{Introduction}

Ixeris sonchifoia (Bge.) Hance is a plant of the Compositae family, which is also known as "Kudiezi", "Mantianxing", etc. I. sonchifoia is a biennial herb mainly distributed in northeast China, north China and Inner mongolian. ${ }^{[1]}$ I. sonchifoia is listed as the top species with the effects of clearing away heat-evil, alleviating pain eliminating swelling, and for the treatment of appendicitis, enteritis, dysentery and various purulent inflammation according to Shennong Herbal Classic and Inner Mongolian Chinese Herbal Medicine. ${ }^{[1]}$ Modern phytochemical and pharmacological investigation revealed the anti-tumor, anti-inflammatory, analgesic, sedative and cardiovascular diseases activities of this $\mathrm{TCH}$. Recent studies have shown that it can also inhibit platelet aggregation, increase fibrinolytic enzyme activity, increase coronary flow, improve myocardial circulation, and have inhibitory effects on ascites, liver cancer and cervical cancer. I. sonchifoia showed valuable medicinal value with many advantages such as abundant source, low price, wide and exact curative effect, no toxic side effect, etc. This review summarized the phytochemical and pharmacological progress on $I$. sonchifoia to provide a reference for better understanding its effective substances.

\section{Chemical Constituents}

Since the 1980s, researchers have conducted a series of studies on the chemical constituents of $I$. sonchifoia. More than 100 compounds involving flavornoids, triterpenoids, sesquiterpene lactones and other compounds have been isolated from this plant. ${ }^{[3-6]}$

\section{Flavornoids}

The content of flavonoids in $I$. sonchifoia is high, and 15 species have been found with flavoids (Figure 1). According to the structures of the flavonoid aglycones, the flavonoids in $I$. sonchifoia can be classified into two types: luteolinv (1) type and apigenin (2) type. ${ }^{[7]}$ Eight luteolin glycosides, luteolin-7$O-\beta-D$-glucopyranoside (3), luteolin-7-O- $\beta$ - $D$-glucuronopyrano- side methyl ester (4), luteolin-7-O- $\beta$ - $D$-glucuronopyranoside ethyl ester $(5),{ }^{[2,8,9]}$ lutein-7-O-gentibioside (6) and luteolin-7-O$\beta$-D-glucuronopyranoside (7) and luteolin-7-O- $\beta$ - $D$-glucopyranosyl- $(1 \rightarrow 2)-\beta$ - $D$-glucopyranoside $(8)^{[10]}$ and four apigenin-glycosides, apigenin-7-O- $\beta$ - $D$-glucopyranoside (9), apigenin-7-O- $\beta$ $D$-glucuronopyranoside methyl ester (10) and apigenin-7-O$\beta$-D-glucuronopyranoside (11). ${ }^{[1]}$ Among them, luteolin-7-O$\beta$ - $D$-glucuronopyranosideis as the main active ingredient and content is the highest in $I$. sonchifoia.<smiles>[R9]c1cc(O)c2c(c1)Oc1cc(O)c([R])cc1O2</smiles><smiles></smiles>
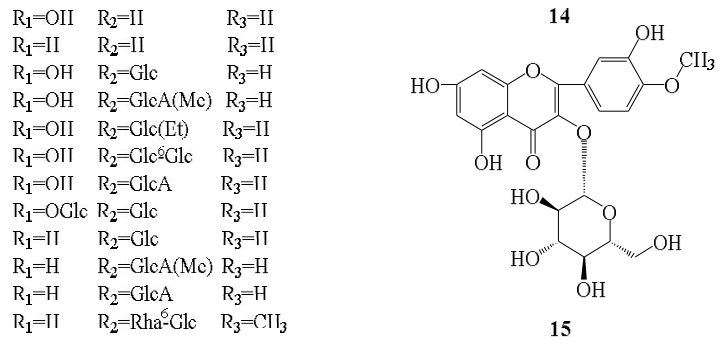

Figure 1 Flavonoids isolated from $I$. sonchifoia.

Besides the above mentioned constituents, four other flavonoids constituents were also isolated, namely 5,7-dihydroxy4 '-methoxy-flavone-7-O-rutinoside (12), ${ }^{[3]}$ quercetin (13), rutin (14) and isorhamnetin-3-O- $\beta$ - $D$-glucoside (15). ${ }^{[11,12]}$

\section{Triterpenoids}

In addition to flavonoids, triterpenoids are another type of characteristic constituents in I. sonchifoia. 16 triterpenoids in- 
cluding skeleton of oleane, ursane, dandelion and lupin ${ }^{[6]}$ were found in I. sonchifoia (Figure 2).
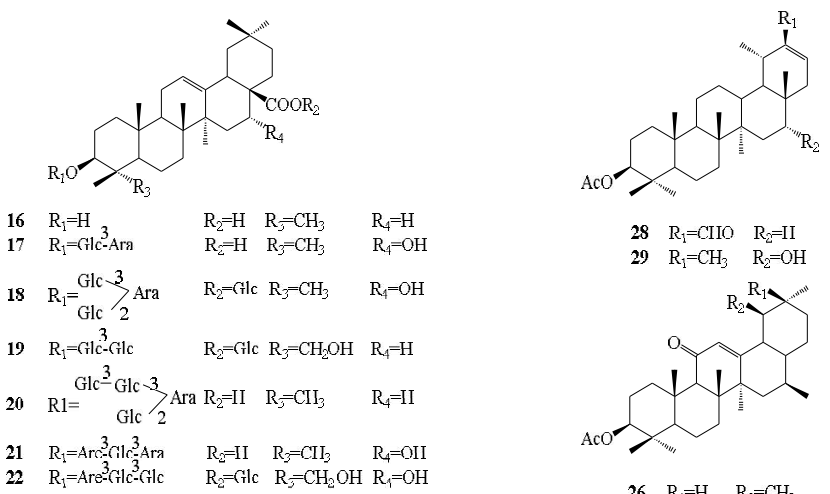

$28 \mathrm{R}_{1}=\mathrm{CIO} \quad \mathrm{R}_{\mathrm{c}}=\mathrm{II}$

$$
29 \mathrm{R}_{1}=\mathrm{CH}_{3} \quad \mathrm{R}_{2}=\mathrm{OH}
$$

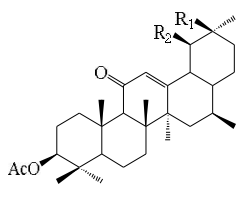

$26 \quad \mathrm{R}_{1}=\mathrm{H} \quad \mathrm{R}_{2}=\mathrm{CH}_{3}$

$27 \mathrm{R}_{1}=\mathrm{CH}_{3} \mathrm{R}=\mathrm{H}$
23

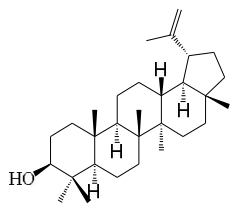

30

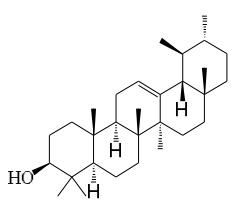

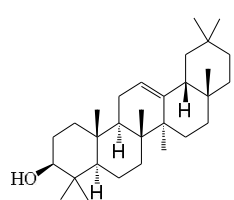

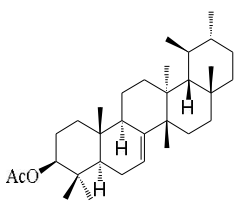

31

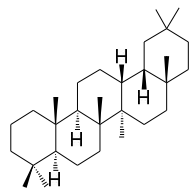

25

Figure 2 Triterpenoids isolated from I. sonchifoia.

These triterpenoids were identified asoleanolic acid (16), ${ }^{[13]}$ ixeris saponins A-F (17-22), ${ }^{[14]} \alpha$-amyrin (23), $\beta$-amyrin (24), oleanane (25), ${ }^{[15]} 3 \beta$-hydroxyurs-12-en-11-one acetate (26), $3 \beta$-hydroxyolean-12-en-11-oneacetate $(27), \quad 3 \beta$-acetoxytaraxastane-20-en-30-al (28), taraxaster-20-en-3 $\beta$, 16 $\alpha$-diol-3acetate (29), lupeol $(30)^{[2,14]}$ and bauereny lacetate (31). ${ }^{[13]}$

\section{Sesquiterpenes lactones}

Sesquiterpenes are the characteristic consituents in $I$. sonchifoia, and there are 17 compounds with defined structures (Figure 3). According to their skeletons, the sesquiterpenes lactones in I. sonchifoia can be classified into two types: guaianolide-type and germacranolide-type. Sonchifolactones A-D (32-35) were isolated from the chloroform extraction layer. $^{[2]}$ 8-Desoxyartelin (36), ixerin $X(37)$, ixerin $Z$ (38), $9 \alpha$-hydroxyzaluzlin (39), ${ }^{[14]}$ 8-desoxyartelin- $\beta$ - $D$-glucopyranoside (40), ixrinoside $A(41),{ }^{[16]}$ and sonchifoliasolides $A-H$ $(42-49)^{[17,18]}$ were also isolated from I. sonchifoia.

\section{Phenylpropanoids}

\section{Lignans}

Lignans is a type of natural products of oxidative polymerization of phenylpropanoids, mostly in the form of dimers, and a few are trimers and tetramers. There are six lignans isolated from the $I$. sonchifoia (Figure 4), named asdihydroconiferol $(50),{ }^{[19]}$ sonchifolignan $A(51)$, sonchifo-lignan $B(52),{ }^{[2,14]}$ sonchifolinin $(53),{ }^{[20]}(+)$-syringarensinol $(54)^{[6]}$ and glochidioboside $(\mathbf{5 5}),{ }^{[21]}$ respectively.

\section{Coumarins}

Lu et al. isolated esculetin $(56)^{[15]}$ from this plant for the first time (Figure 4).

\section{Adenosine}

In 1981, Meng et al. isolated and identified adenine nucleosides $(57)^{[5]}$ in the whole grass of $I$. sonchifoia, which showed an effect of expanding coronary artery.
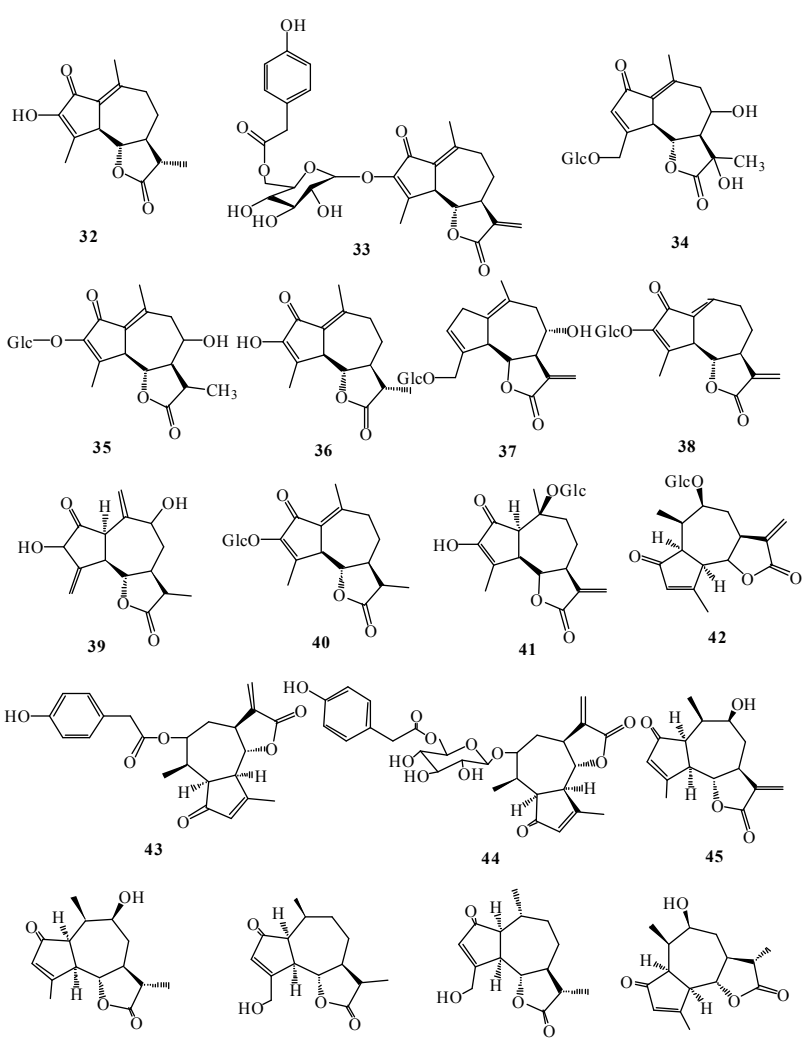

46

47

Figure 3 Sesquiterpenes lactones isolated from I. sonchifoia.
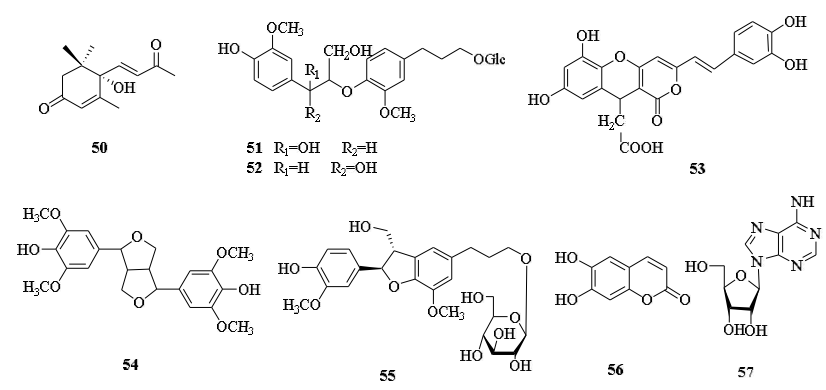

Figure 4 Phenylpropanoids and adenosine isolated from $I$. sonchifoia.

\section{Sterols}

Three types of compounds including $\beta$-sitosterol (58), daucosterol $(59)^{[13]}$ and stigmasterol $(\mathbf{6 0})^{[22]}$ were isolated from 1 . sonchifoia (Figure 5).

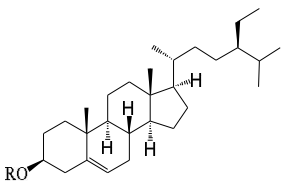

$\begin{array}{ll}58 & \mathrm{R}=\mathrm{H} \\ 59 \mathrm{R}=\mathrm{gl} \text {. } & \end{array}$

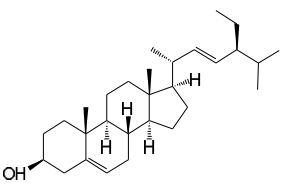

60

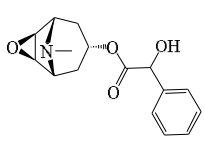

61
Figure 5 Sterols and alkaloids isolated from I. sonchifoia.

\section{Alkaloids}

Ma et al. isolated scopolamine $(61)^{[8]}$ from $I$. sonchifoia (Figure 5).

\section{Organic acids and fatty acids}

$(E)-2,5$-Dihydroxycinnamic acid (62), ${ }^{[8]}$ vanillic acid $(63)^{[24]}$ and ferulic acid $(64)^{[14]}$ were isolated from $I$. sonchifoia (Figure 6 ). Moreover, others were found in this plant, named as palmitic 
acid, succinic acid, linoleic acid ${ }^{[3,15]}$ chicoric acid, (-)-3,4-dihydroxycaffeoyl tartaric acid, ${ }^{[10]}$ 1-furancarboxylic acid, ${ }^{[20]}$ dandelion- $B,{ }^{[25]}$ hydroxybenzoic acid, ${ }^{[24]}$ caffeic acid, original acid and 3,4-dihydroxybenzoic acid. ${ }^{[7]}$<smiles>O=C(O)CCc1cc(O)ccc1O</smiles>

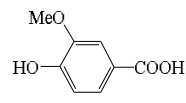

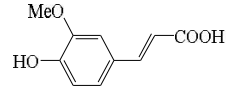

64
Figure 6 Organic acids and fatty acids isolated from I. sonchifoia.

\section{Amino acids}

A variety of amino acids such as glutamic acid, aspartic acid, serine, proline, citric acid and arginine were isolated and identified from $I$. sonchifoia by Young et al. ${ }^{[26]}$

\section{Other compounds}

Other types of compounds were isolated from I. sonchifoia named as dehydrovomifoliol (65), loliolide (66), ${ }^{[19]}$ 1,3-dihydroxypyrimidine (67), ${ }^{[20]}$ 3,4,5-trimethoxyphenyl-1$O-\beta$ - $D$-glucopyranoside (68), ${ }^{21]}$ (Figure 7) $3 \beta$-acetoxy-11oxou-rs-12-ene, $3 \beta$-acetoxy-20-tamxasten-22-one, ${ }^{[13]} 3 \beta$ hydroxy-olean-12-en-11-one acetate, 3,4-dihydroxybenzalacetone ${ }^{[3]}$ 8-deoxyartemisactone, ${ }^{[13]}$ protocatechuic acid ethyl ester, vanillin, protocatechuic aldehyde, 3-(4'-hydroxy-3'methoxy-phenyl)-1-propanol, lirioresinol-A, ${ }^{[19]}$ wax alcohol, tryptamine, 11 $\beta, 13$-dihydrosantamarine, $3 \beta$-hydroxy-11 $\beta, 13-$ dihydrocostu-nolide, ixerisoside $E$, 8-epidesacylcinaropicrin glucoside, ${ }^{[15]}$ bis-(2-ethylhexyl)phthalate, $p$-hydroxybenzaldehyde, 1,4-ben-zenedimethanol, and echinocystic acid-3-O$\beta$ - $D$-glucopyran-osyl-( $(\rightarrow 3)-\alpha$ - $L$-arabinopyanoside.<smiles>CCC(O)C=CC1(C)C(C)CC(O)CC1C</smiles>

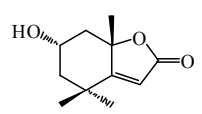

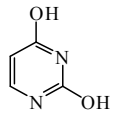

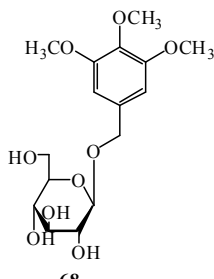

68

Figure 7 Other compounds isolated from I. sonchifoia.

In addition, Qi et al. identified 37 compounds from the volatile oil components of $I$. sonchifoia, including fatty alkanes, ketones, aldehydes, alkenes, enols, alkenes and esters. The main component of the volatile oil is 6,10,14-trimethyl-2pentadecanone. ${ }^{[26]}$

\section{Biological activities and clinical applications}

Pharmacological study on $I$. sonchifoia revealed that the crude extracts and pure compounds showed a wide range of biological activities, including the treatment of cardiovascular and cerebrovascular diseases, and anti-tumor, analgesic and sedative activities. "Kudiezi injection" made by its active ingredient is clinically used for treating diseases such as coronary heart disease.

\section{Activity for cardiovascular and cerebrovascular diseases}

The adenosine and flavonoids are potent ingredients to treat cardiovascular disease. ${ }^{[27-31]} \mathrm{Hu}$ et al. reported $I$. sonchifoia has a preventive effect on atherosclerosis $(P<$ $0.01)^{[31]} \mathrm{Jia}$ et al. found 16 and $8 \mathrm{mg} / \mathrm{kg}$ group of flavonoids of Kudiezi could reduce the mortality of mice, prolonged death time $(P<0.05, P<0.01) ; 16,8,4 \mathrm{mg} / \mathrm{kg}$ group could improve behavioral disorder and reduce behavioral score $(P<0.05, P<$ $0.01) ; 10,5 \mathrm{mg} / \mathrm{kg}$ group could reduce the extent of cerebral infarction in rats with focal cerebral ischemia, reduce the water

content of brain tissue and the content of nitric oxide synthase and inducible nitric oxide synthasem $(P<0.05)$. It indicated the flavonoids of $I$. sonchifoia could protect ischemic brain injury. ${ }^{[28]}$ Wang et al. found Kudiezi injection showed a significant protective effect on ischemic myocardium. ${ }^{[32]}$ Feng et al. proved the extract of Kudiezi could treat coronary heart disease. ${ }^{[33]}$ Tao et al. reported Kudiezi injection showed a lipid-lowering effect on patients with unstable angina pectoris and further relieved clinical symptoms, improved myocardial ischemia and reduced the incidence of angina pectoris. ${ }^{[34]}$ Zhao et al. reported $I$. sonchifoia could effectively relieve the symptoms of coronary heart disease and angina pectoris. ${ }^{[35]}$

\section{Anti-tumor activity}

Sesquiterpene lactones and triterpenoids from I. sonchifoia are active components foranti-tumor. Liu et al. found $l$. sonchifoia could inhibit the growth of transplanted tumors in animals. The inhibition rate of transplanted sarcoma 180 (S180) in high, medium and low dose groups were $39.86 \%, 38.14 \%$, $35.73 \%$, respectively. It is also found $I$. sonchifoia could prolong the survival time of mice with Heric's ascites tumor. The prolongation rate of mice in high, medium and low dose groups were $41.27 \%, 29.78 \%, 17.88 \%$, respectively. ${ }^{[36]}$ Feng et al. reported saponin $B$ and $C$ showed obvious cytotoxicity effects on cancer cell lines A375 (human melanoma cells), L929 (mouse lung epithelial cells) and HeLa (human cervical cancer cells) with $\mathrm{IC}_{50}$ values ranging from 8.83 to $15.83 \mu \mathrm{M} .{ }^{[14]}$ Wang et al. observed that $I$. sonchifoia inhibited the growth of melanoma cells in a concentration-dependent manner. ${ }^{[37]}$ Zhou et al. found Kudiezi injection $(5,10,20 \mathrm{mg} / \mathrm{kg}$ ) showed a significant inhibitory effect on tumor growth in three tumor-bearing mice $(P$ $<0.05, P<0.01, P<0.001)$. At the same time, it increased significantly the number of peripheral blood leukocytes in liver cancer $\mathrm{H} 22$ mice $(P<0.05)$. It could also inhibit the proliferation of human cervical cancer Hela cells or liver cancer HepG-2 cells in vitro. ${ }^{[38]}$

\section{Analgesic and sedative activity}

Feng et al. found that Kudiezi injection showed an analgesic and sedative effect. ${ }^{[3,39]}$

\section{Activity of regulating blood lipids}

Zhao et al. proved that saponin $(400 \mathrm{mg} / \mathrm{kg}$ ) showed an anti-atherosclerosis effect by regulating blood lipid metabolism and correcting free radical metabolism disorders. ${ }^{[40]} \mathrm{Li}$ et al. found that Kudiezi injection could significantly regulate the blood lipid metabolism in experimental hyperlipidemia rats. ${ }^{[2]}$ Sohn et al. reported $I$. sonchifoia could reduce the increase of serum total cholesterol, triglyceride, low-density lipoprotein cholesterol and glutamate oxaloacetate transaminase in rats, which was caused by ethanol. ${ }^{[41]}$

\section{Anti-oxidation activity}

Zhao et al. reported that the flavonoids and lignans showed strong activity of scavenging free radical and the potential to become natural antioxidant. ${ }^{[21]}$

\section{Antiviral effect activity}

Liu et al. reported that Kudiezi showed a good inhibitory effect on respiratory influenza virus $\mathrm{H}_{3} \mathrm{~N}_{2}$, syncytial virus (RSV) and adenovirus (ADV-3). ${ }^{[36]}$

\section{Liver protection activity}

Zhang et al. reported that Kudiezi injection showed a certain anti-fibrosis effect. ${ }^{[42]}$ Zhao et al. found Kudiezi injection showed a significant therapeutic effect for the liver fibrosis. ${ }^{[43]}$ Guo et al. confirmed Kudiezi injection had a good therapeutic effect for rats with acute alcoholic liver injury. ${ }^{[44]}$ 


\section{Activity of improving blood system}

Wei et al. found that Kudiezi injection showed an effect of improving blood circulation and microcirculation. ${ }^{[45]}$ Wei et al. have proved $I$. sonchifoia could prevent and treat diabetic vascular lesions by lowering blood fat and blood viscosity. ${ }^{[46]}$ Feng et al. found that $I$. sonchifoia could inhibit platelet aggregation significantly in the blood system and increase the activity of plasmin significantly, which can treat thromboembolic disease ${ }^{[47]}$ Zhang et al. found Kudiezi could dilate blood vessels, improve microcirculation, reduce vascular resistance, increase blood flow, reduce platelet aggregation, inhibit thrombosis and reduce plasma viscosity. Thereby, it could reduce the occurrence, development and improvement of ischemia and hypoxia. ${ }^{[48]}$

\section{Other activities}

Bae et al. found that the extract of $I$. sonchifoia showed an effect of increasing the stability and fluidity of phospholipid liposomes. ${ }^{[49]}$ Tong et al. reported Kudiezi had a protective effect on rat kidney caused by anti-oxidative stress. ${ }^{[50]}$ Wu et al. found $I$. sonchifoia could increase the expression of vascular endothelial growth factor, promote capillary angiogenesis, reduce inflammatory infiltration of the flap, and improve the survival rate of rat random flaps significantly. ${ }^{[51]}$ Peng et al. proved $I$. sonchifoia could promote the proliferation of HUVEC cells $(P<0.05){ }^{[52]}$

\section{Conclusion and Perspective}

I. sonchifoia is a famous Chinese traditional herb with wide distribution, rich resources and variously clinical use. Kudiezi injection is widely used for treating cardiovascular and cerebrovascular diseases. Compared with its chemical compositions and clinical applications, the pharmacological research is not systematic and comprehensive enough, and it needs further investigation. Therefore, comprehensive research on pharmacological effects should be strengthened to provide more direct guidance for clinical applications, especially in pharmacological mechanisms, target research, and medical combination.

\section{Acknowledgement}

This work was supported by the Science and Technology Tackling Project of Henan Science and Technology Depratment (No. 182102310063).

\section{References}

[1] Yuan, T.; Liu, R.; Huang, X. L. Pract. Pharm. Clin. Remed. 2004, 7, 44.

[2] Feng, X. Ph.D. Dissertation, Shenyang Pharmaceutical University, Shenyang, China, 2001.

[3] Hou, J.; Ai, R. T.; Zhou, L. Y.; Zhou, L. Y.; Liu, B. J. J. Shenyang Pharm. Univ. 2011, 28, 879.

[4] Ye, G.; Fan, M. S.; Huang, C. G.; Qu, D. Y. Chin. Pharmacol. 2005, 40,1613

[5] Meng, X. Z.; Ni, S. F.; Su, H. X. Chin. Tradit. Herbal Drugs 1981, 12, 4.

[6] Yang, Z.; Li, X. L.; Shi, L. G.; Yang, S. J Qiqihar Univ. 2012, $28,52$.

[7] Ma, J. Y.; Wang, Z. T.; Xu, L. S; Xu, G. J.; Wang, Y. X. J. Chin. Pharm. Univ. 1998, $29,94$.

[8] Feng, X. Z.; Xu, S. X.; Dong, M. Chin. Pharm. J. 2000, 9, 134

[9] Jiang, R. Z.; Chen, Y. H.; Luo, H. M.; Xu, D. D.; Gao, Y. Nat. Prod. Res. Dev. 2010, 2, 430.

[10] Bai, X. L. Chin. Mod. Med. 2016, 23, 4.

[11] Wang, Z. G. Ph.D. Dissertation, Shenyang Pharmaceutical University, Shenyang, China, 2006.

[12] Zhang, M. D. Ph.D. Dissertation, Shenyang Pharmaceutical University, Shenyang, China, 2005.

[13] Ye, G.; Fan, M. S.; Huang, C. G. Chin. Pharmacol. J. 2005, 40,
1613.

[14] Feng, X. Z.; Xu, S. X.; Yao, J. P.; Sha, Y.; Li, W. J. Chin. Tradit. Herbal Drugs 2001, 32, 799.

[15] Lu, Y.; Zhang, C. F.; Zhang, M. Chin. Pharm. J. 2007, 16, 214.

[16] Suh, J.; Jo, Y.; Deuk kim, N.; Ja Bae, S.; Jung, J. H.; Silk Im, K. Arch. Pharm. Res. 2002, 25, 289.

[17] Zhang, W. Z.; Li, X. L.; Shi, L. G.; Wang, J. L.; Zhao, M. J. Asian Nat. Prod. Res. 2009, 11, 1087.

[18] Zhang, W. Z.; Li, X. L.; Wang, M. J.; Shi, L. G.; Zhao, D. F. J. Asian Nat. Prod. Res. 2008, 10, 753.

[19] Zhang, N.; Lv, A. L.; Wang, D.; Cheng, G.; Dang, Q. J. Shenyang Pharm. Univ. 2007, 24, 54.

[20] Huang, H. Ph.D. Dissertation, Jilin University, Jilin, China, 2006.

[21] Zhao, C. Y. J. Pharm. Pract. 2016, 34, 24.

[22] Dai, J. N; Yin, R.; Chen, X. H; Bi, K. S. Northwest Pharm. J. 2006, $21,94$.

[23] Sun, L.; Yan, Y. B. Chin. J. New Drugs 2011, 20, 2460.

[24] Li, Y, Y; Yi, Y, Z; Sui, D. D.; Yu, X. F.; Qu, S. F. J. Jilin Univ. 2008, 28 , 357.

[25] Young, H. K. Hanguk Yongyang Hakhoechi 1992, 231, 296.

[26] Qi, X. L.; Meng, X. Y.; Wang, S. P.; Wu, Y.; Bao, Y. L. Mol. Sci. 2006, $22,138$.

[27] Jia, D. W.; Luo, S. Y.; Ye, S. S.; Jia, D. Y.; Li, X. W. Chin. J. Clin. Pharm. Therap. 2011, 16, 616.

[28] Chen, C. G.; Jia, H. L.; Lv, S. X.; Xu, C. Q. Chin. J. Clin. Pharmacol. 2012, 28, 196.

[29] Li, Y. Y.; Yin, Y. Z.; Pei, D. Y.; Yu, X. F.; Qu, S. C. Jilin Univ. Med. Sci. 2002, 28, 357.

[30] Zai, Y. R.; Hang, X. N.; Yu, X. F.; Qu, S. C.; Sui, D. Y. Pharmacol. Clin. Chin. Mater. Clin. Med. 2008, 24, 53.

[31] Hu, D. Q; Sun, Q.; Zhang, J. J; Song, G. Y. Chin. Med. Herald 2013, $10,23$.

[32] Xin, T. G. Chin. Mod. Doctor 2010, 48, 45.

[33] Feng, Y. S.; Gui, L. H.; Wei, W. Z. Chin. Tradit. Herbal Drugs 1979 , $10,31$.

[34] Tao, G.; Li, Y. J. Guangdong Med. 2010, 31, 910.

[35] Zhao, Q.; Ye, W. B. Asia-Pacific Tradit. Med. 2011, 7, 123.

[36] Liu, X. L.; He, Y. H.; Jin, Y. S.; Liu, Y.; Jiang, C. Y. Mod. Chin. Med. 2006, 8, 11.

[37] Wang, J. G.; Jiang, Z.; Xie, Z. Prog. Anat. Sci. 2013, 19, 148

[38] Zhou, X. M.; Zheng, H. H.; Cao, C. Y. J. Shenyang Pharm. Univ. 2007, 24, 103.

[39] Ma, H.; Sha, M. J. Liaoning Univ. Tradit. Chin. Med. 2005, 7, 224.

[40] Zhao, Y. W.; Xie, W. L.; Yu, X. F.; Qu, S. C.; Sui, D. Y. Med. J. Chin. People's Armed Police Force 2005, 14, 165.

[41] Sohn, H. S. Hanguk Yongyang Hakhoechi 2001, 34, 493.

[42] Zhang, H. X.; Liu, F.; Ge, Q. L. Clin. J. Med. Officer 2011, 39, 1052.

[43] Zhao, J. H.; Wu, S. G.; Wu, Q. S.; Guo, K. N.; Dong, X. J. Lishizhen Med. Mater. Med. Res. 2010, 21, 586.

[44] Guo, K. N.; Qian, N.; Dong, X. J.; Wu, S. G. Lishizhen Med. Mater. Med. Res. 2009, 20, 543.

[45] Wei, R.; Lin, G. X. J. Guiyang Coll. Tradit. Chin. Med. 2001, 23, 59

[46] Wei, G. L. Liaoning J. Pract. Diabetes 2002, 10, 28.

[47] Feng, Y. S.; Gui, L. H.; Wei, W. Z. Acad. J. Shenyang Coll. Pharm. $1981,13,23$

[48] Zhang, H. W.; Cheng, F.; Yan, Q. S. Chin. J. Conval. Med. 2001, 10, 21.

[49] Bae, S. J.; Roh, S. B.; Jung, B, M. Hanguk Yongyang Hoechi 1998, 27, 508.

[50] Tong, S. D.; Shi, G. H.; Lu, H. Y. Shanxi Chin. J. Chin. Med. 2013 34, 618.

[51] Wu, S. M.; Liu, Y. B.; Lin, D. S. Chin. J. Orthopa. Trauma 2013, 26, 689.

[52] Peng, Y. H.; Zeng, H. L.; Jiang, H.; Cai, Y.; Chen, M. J. North Sichuan Med. Coll. 2011, 26, 480.

Received March 19, 2019 Accepted May 8, 2019 